\title{
ATUAÇÃO DO FARMACÊUTICO NA SAÚDE ESTÉTICA
}

\author{
THE PERFORMANCE OF THE PHARMACIST IN HEALTH BEAUTY \\ Caroline de Paula Luiz ${ }^{1}$ \\ Luciana Colli
}

RESUMO: A área farmacêutica é bastante ampla e após a mudança das diretrizes curriculares surgiram novas áreas onde o farmacêutico estaria apto para atuar, dentre elas a estética. O Conselho Federal de Farmácia - CFF através da resolução 573/2013 conhece a saúde estética como um novo campo de atuação para os farmacêuticos, assim dando o titulo de Farmacêutico esteta, tornando-lhe responsável pela aquisição das substâncias e dos equipamentos necessários para o desenvolvimento e aplicação das técnicas. O CFF elaborou e aprovou duas novas resoluções: a 616/2015 que atribui ao Farmacêutico a atuação no âmbito da estética passando a ampliar suas técnicas nesta área de atuação assim como, os recursos terapêuticos utilizados por esse profissional e a 645/2017, ampliando o rol de atividades, legitimando e dando força total para a nova profissão. O presente estudo foi elaborado para relatar a atuação do farmacêutico na "Saúde Estética" abordando recursos terapêuticos utilizados por este profissional, mostrando as habilidades de atuação para a realização de procedimentos estéticos com os devidos cuidados para garantir a segurança e a saúde do paciente.

Palavras-chave: Atenção Farmacêutica. Farmacêutico. Estética. Saúde estética.

ABSTRACT: The pharmaceutical area is quite wide and after the change of curriculum guidelines new areas emerged where the pharmacist would be able to act, among them aesthetics. The Federal Pharmacy Council - CFF through resolution $573 / 2013$ knows aesthetic health as a new field of action for pharmacists, thus giving the title of Aesthete Pharmacist, making it responsible for the acquisition of the substances and equipment necessary for the development and application of techniques. The CFF elaborated approved two new resolutions: 616/2015 which attributes to the Pharmacist the performance in the field of aesthetics, expanding its techniques in this area of activity as well as the therapeutic resources used by this professional and 645/2017, expanding the list of activities, legitimizing and giving full force to the new profession. The present study was developed to report the pharmacist's performance in "Aesthetic Health" addressing therapeutic resources used by this professional, showing the skills of action to perform aesthetic procedures with proper care to ensure the safety and and health of the patient.

\footnotetext{
${ }^{I}$ Formanda em farmácia . Universidade Iguaçu- Unig. E-mail: carolpaular998@gmail.com.

2 Orientadora. Farmacêutica .
} 
Keywords: Pharmaceutical care. aesthetics. Pharmacist. Aesthetic health.

\section{INTRODUÇÃO}

A imagem pessoal tem estimulado uma grande procura por recursos estéticos em busca de uma melhoria na aparência. Os padrões de beleza sofreram grandes mudanças que conduziram uma busca maior pela recuperação e aprovação social através de procedimentos estéticos. A busca pela jovialidade estimula o ser humano a recorrer ao mundo da estética, evidenciando um aumento deste campo nos últimos anos e destacando-se por um número cada vez mais significativo de práticas e técnicas para cuidar da beleza.

O processo de envelhecimento e o corpo fora do padrão estipulado principalmente pela mídia não são mais vistos como um processo natural na vida, e sim algo que pode ser curado, como uma doença, estimulando o grande consumo de produtos estéticos e procedimentos cirúrgicos. (LEAL et al., 2007).

A indústria está desenvolvendo produtos cada vez mais avançados que podem amenizar problemas estéticos e com dois importantes diferenciais que correspondem ao menor custo e tempo de recuperação. Os pacientes estão procurando cada vez mais tratamentos menos invasivos que tenham menor tempo de recuperação (HIRATUKA, 2008).

O baixo custo em relação a uma cirurgia plástica também tem sido um fator diferencial no aumento da busca de profissionais capacitados para utilização dos recursos estéticos.

A Organização Mundial de Saúde (OMS) define saúde com a ausência de doença e uma condição de perfeito bem-estar físico, mental e social. Os procedimentos estéticos associados a técnicas de massagem e terapias alternativas contribuem com estes objetivos, através do emprego de cosméticos e equipamentos específicos, respeitando as características e anseios do paciente. Farmácia estética tem crescido muito e o farmacêutico habilitado estará apto a se responsabilizar por estabelecimentos de saúde estética, a realizar procedimentos não invasivos e invasivos não cirúrgicos, utilizando recursos terapêuticos estéticos e realizando o uso e compras de produtos e equipamentos utilizados nesses procedimentos. 
O Farmacêutico é um profissional de destaque, pois possui conhecimentos que garantem sua atuação em várias áreas, como a estética, utilizando seu conhecimento científico e intelectual, agregados à ética e atenção farmacêutica e as atividades de práticas clínicas. (FERREIRA, 2016).

O farmacêutico que tem o título de esteta atua de acordo com as resoluções que respaldam a "Saúde Estética", as quais o permitem utilizar recursos terapêuticos estéticos, realizando a avaliação e aconselhamento quanto ao uso da: cosmetoterapia; peelings químicos e mecânicos; sonoforese; eletroterapia; iontoforese; radiofrequência estética; criolipólise; luz intensa pulsada; laserterapia; carboxiterapia; agulhamento e microagulhamento, toxina botulínica; preenchimentos dérmicos; intradermotepia; laserterapia ablativa e fio lifting de auto sustentação. (BRASIL, 2016a; BRASIL 2017).

Este breve estudo tem como objetivo apresentar a atuação do farmacêutico na saúde estética, abordando alguns dos recursos terapêuticos utilizados por esses profissionais, de acordo com a legislação, destacando a importância da atenção farmacêutica e qualificação deste profissional para atender na área esteta.

\section{OBJETIVOS}

\section{2.r Objetivo geral}

- Esta pesquisa tem como objetivo relatar sobre o farmacêutico na saúde estética, as áreas de atuação, os métodos e procedimentos utilizados e que podem ser atuado pelo farmacêutico, e como a área tem se tornado um grande alvo entre as pessoas em busca da imagem perfeita.

2.2 Objetivos específicos

- Visualizar o farmacêutico como profissional esteta

- Abordar sobre a atenção farmacêutica na estética

- Citar as legislações vigentes que permitem a atuação do farmacêutico na saúde estética

- Analisar os procedimentos estéticos que o farmacêutico pode atuar

- Descrever os benefícios de alguns procedimentos estéticos. 


\section{METODOLOGIA}

O estudo trata-se de uma revisão bibliográfica, sendo a busca realizada através de revistas e artigos científicos (português e inglês) especializados na área de farmácia estética, publicados pelas seguintes plataformas: Scientific Eletronic Library Online (SCIELO), Google Acadêmico, Sites do Conselho Federal de Farmácia (CFF) e Conselhos Regionais de Farmácia (CRF's), no período de julho a novembro de 202I, onde abordasse a atuação do farmacêutico na saúde estética com base nas resoluções do conselho federal de farmácia que estabelece as atribuições do Farmacêutico Esteta, dentre os procedimentos invasivos não cirúrgicos que o mesmo pode atuar.

\section{DESENVOLVIMENTO}

\section{I Saúde estética}

O conceito do corpo perfeito pode abranger diversos aspectos, desde algo real até o imaginário. (CAMARGO et al., 20II). A evolução fisiológica do corpo humano tem ocasionado muitas angustias e preocupações, principalmente nas mulheres. $\mathrm{O}$ fator envelhecimento é o que vem assustando mulheres da terceira idade e jovens precocemente, pois mexe diretamente com a aparência física, causando um grande aumento nas buscas por clínicas estéticas para melhorar ou amenizar características indesejadas.

A estética atrai a atenção de todas as camadas sociais, de pessoas de diferentes níveis culturais e econômicos, e os produtos variam de preço e atendem as mais distintas finalidades. Daí a importância da qualificação profissional. É imprescindível conhecer as técnicas, estar bem informada e estabelecer tratamentos que inspirem segurança e garantam eficácia. (ALMA; COSTA, 20II).

Nas atuais pesquisas sobre corpo, observam-se frequentemente estudos relacionando saúde com estética. Verificando crescentes problemas na sociedade atual, associados ao binômio saúde-estética, justificados pela indução da mídia, em especial a televisão e as revistas, produzindo e ditando padrões de beleza e de saúde (CAMARGO et.al., 2oII). Vários estudos abordam os aspectos de conceituação e descrição sobre formas e técnicas de tratamentos inovadores relacionados à saúde 
estética. O farmacêutico que se insere nessa área deve acompanhar o crescimento da beleza mundial. (SOUZA, 2012; BARROS; OLIVEIRA, 2017).

A Saúde Estética é a área da saúde voltada à promoção, proteção, manutenção e recuperação estética do indivíduo, de forma a selecionar, aplicar procedimentos e recursos estéticos, utilizando-se para isto produtos cosméticos, técnicas e equipamentos específicos, de acordo com as características e necessidades do cliente (BRASIL, 2013)

\subsection{Farmacêutico esteta}

A formação do farmacêutico abrange o conhecimento em várias áreas básicas e especificas dando ao farmacêutico a possibilidade de orientar e indicar aos pacientes suplementos alimentares e nutracêuticos, com a finalidade de intensificar o tratamento estético. Isso o habilita a atuar nas áreas de medicamentos, desempenhar ato de assistência farmacêutica na área de cosmetologia e em procedimentos na saúde estética, anatomofisiologia da pele, disfunções estéticas, procedimentos estéticos, entre outros, e ainda conhecer necessidades e determinar cuidados para promover a saúde e a qualidade de vida dos pacientes (BECKER, 2015). As diretrizes de atuação do farmacêutico no âmbito da estética estabelecem que o egresso deva estar devidamente capacitado ao exercício das atividades que envolvem a profissão e deve se compor de um perfil de princípios éticos para que possa compreender a sociedade em seu segmento social, cultural e econômica, conduzindo sua atuação para oferecer serviços de qualidade. (SERAFIN; CORREIA JUNIOR; VARGAS, 2015).

Para GADELHA (2003) o uso das técnicas mais modernas de tratamento estético proporciona resultados melhores com o passar dos anos, melhorando o nível de qualidade de vida.

Mateus (2017) relata uma migração de cirurgias plásticas para tratamentos estéticos, por serem procedimentos de fácil excesso e baixo custo, o mercado da estética requer constantes inovações, assim como mão de obra qualificada e especializada, para que possa atender a demanda que vem aumentando a cada dia. 


\subsection{Atenção farmacêutica}

O farmacêutico deverá ser qualificado do ponto de vista técnico, científico e profissionalizante para o uso de técnicas e habilidades terapêutico de natureza estética (BECKER, 2015). Brasil (2013) diz que o farmacêutico poderá ser o responsável técnico do estabelecimento nos quais se utilizam técnicas de natureza estética e recursos terapêuticos para fins estéticos.

Deve confirmar se o estabelecimento onde assumirá a responsabilidade técnica este legalmente constituído e autorizado para a realização de suas atividades, especialmente junto ao Sistema Nacional de Vigilância Sanitária (SNVS).

\subsection{Legislação da farmácia estética}

As legislações que respaldam o farmacêutico a atuar na estética são:

- Resolução CFF no 573, de 22 de maio de 2013 - "Dispõe sobre as atribuições do farmacêutico no exercício da saúde estética e da responsabilidade técnica por estabelecimentos que executam atividades afins".

- Resolução CFF no 616, de 25 de novembro de 2015 - "Define os requisitos técnicos para o exercício do farmacêutico no âmbito da saúde estética, ampliando o rol das técnicas de natureza estética e recursos terapêuticos utilizados pelo farmacêutico".

- Resolução CFF № 645, de 27 de julho de 2017- "Dá nova redação aos artigos 2을 e $3^{\circ}$ e inclui os anexos VII e VIII da Resolução/CFF no 616/15”.

\subsection{Procedimentos utilizados pelo farmacêutico}

O farmacêutico esteta tem seus trabalhados voltados para proporcionar a saúde e corrigir as disfunções estéticas, utilizando para isso recursos não invasivos que atuem na melhora da qualidade de vida de seus usuários. (Silva; Filoni; Fitz, 2014). Para exercer procedimentos estéticos, o farmacêutico tem que comprovar a capacitação técnica exigida nas normas do CFF, sendo necessário que este profissional tenha requerido o registro com título de especialista no ramo. (Lorenzet et al.,2015) 
O mesmo é fornecido pelo Conselho Regional de Farmácia de sua jurisdição, visto que só serão praticadas as atividades regidas nas resoluções 25 573/2013 e 616/2015 e 645/207. Entre os recursos terapêuticos que o farmacêutico pode exercer, os mais utilizados são:

- Toxina botulínica;

- Carboxiterapia;

- Criolipólise;

- Fios de Sustentação;

- Peeling químico e mecânico;

\subsection{Toxina botulínica}

A toxina botulínica, conhecida como botox é um produto liberado por fermentação pelas bactérias denominada Clostridium Botulinum que age nas terminações nervosas, bloqueando os canais de cálcio, especificamente bloqueando a liberação de acetilcolina. Quando injetada no musculo em dose e local adequado causa desnervação química parcial e redução da contratura sem ocasionar paralisia completa. Após estudos sua ação ganhou destaque na estética na redução de rugas e linhas de expressão, é utilizado de forma injetável apresentando resultados imediatos com duração de 6 semanas a 6 meses (COLHADO; BOEING; ORTEGA, 2009).

\subsubsection{Carboxiterapia}

Carboxiterapia é uma técnica utilizada para procedimentos estéticos que utilizam ainfusão de gás dióxido de carbônico $\left(\mathrm{CO}_{2}\right)$ de forma subcutânea melhorando a circulação e oxigenação tecidual. A infusão é realizada de forma injetável. Seu mecanismo de ação é baseado na promoção da microcirculação vascular do tecido conectivo, promovendo uma vasodilatação e um aumento da drenagem veno-linfática. Essas ações favorecem o fluxo de nutrientes como os das proteínas importantes no processo de remodelação dos componentes damatriz extracelular e para acomodar a migração e reparação tecidual. Todo o processo traz melhorias teciduais relacionadas à celulite, flacidez, estrias, gordura localizada e resultados pré e pós-operatório de 
lipoescultura por exemplo. Por isso é uma técnica que vem ganhando o mercado estético (CORRÊEA et al., 2008).

\subsubsection{Criolipólise}

A criolipólise é considerada como uma "lipoaspiração não invasiva", onde ocorre a redução da camada de gordura pela exposição ao frio, fazendo com que o tecido adiposo entre em contato com placas congeladas. É utilizado um aplicador 30 que dispõe de uma pressão que destrói a gordura, sem danificar outros tecidos. (SILVA; FILONI; FITZ, 2014).

A técnica é realizada com intuito de diminuir a gordura localizada. A morte das células adiposas ocorre através do resfriamento controlado e localizado por um período de 40 a 60 minutos, com temperaturas acima do congelamento (porém abaixo da temperatura corporal normal). Ao ocorrer o congelamento os lipídeos encontrados dentro do citoplasma dos adipócitos são cristalizados causando apoptose das células e levando a um processo de digestão controlada provocado pelos macrófagos que são responsáveis pela eliminação das células lesadas sem causar danos ao microambiente celular (BORGES; SCORZA, 2014).

\subsubsection{Fios de Sustentação}

Fios de sustentação são técnicas não cirúrgicas para o rejuvenescimento facial. A diminuição da espessura das camadas superficiais e profundas. Da quantidade de vasos da derme e redução da formação de fibras elásticas e colágenas fazem a pele mais fina e flácida emenos nutrida com rugas mais evidentes. Com o entendimento sobre os mecanismos de envelhecimento facial a saúde estética faz uso dos fios de sustentação para suspender pontual eimediatamente a flacidez, estimulando a síntese de colágeno realizando um lifting facial. Atualmente existem os fios de sustentação não absorvíveis e absorvíveis, os absorvíveis são osmais utilizados, pois oferecem maiores vantagens como menores complicações relacionadas aomaterial, tendo uma absorção mais lenta (BORTOLOZO, 2017; BORTOLOZO; BIGARELLA,2016). 


\subsubsection{Peeling químico}

Promove a esfoliação ou remoção das camadas da pele, dependendo do seu objetivo, sempre de modo controlado, a fim de promover uma regeneração dos tecidos epidérmicos e dérmicos, promovendo uma melhoria clínica da pele manchada ou envelhecida (CUNHA, 2014).

Quanto maior a concentração de um ácido e menor o seu $\mathrm{pH}$, mais rápida e profunda é a sua permeabilidade. Vários são os ácidos que podem ser aplicados nos procedimentos de peelings químicos, entretanto os mais utilizados são: glicólico, mandélico, retinóico, salicílico, ascórbico (vitamina C), lático e fenolz (GUERRA et al., 2013)

\subsubsection{Peeling mecânico}

O peeling mecânico é conhecido como microdermoabrasão, é uma técnica realizada no tratamento do rejuvenescimento e da oleosidade facial. A microdermoabrasão é uma técnica de esfoliação não cirúrgica. Esse procedimento elimina toda epiderme e derme papilar, expondo a derme reticular, surgindo uma nova camada epitelial (PINTO, 2012).

\section{CONSIDERAÇÕES FINAIS}

Conclui-se que o farmacêutico especializado em saúde estética é capacitado a realizar procedimentos estéticos, seguindo as orientações do CFF - Conselho Federal de Farmácia através das resoluções vigentes. O farmacêutico é um profissional capacitado para compreende o funcionamento do organismo humano e identificar as necessidades de cada paciente, inclusive estéticas.

A estética vem crescendo cada vez mais na sociedade, com uma grande busca por procedimentos estéticos com aparelhos menos invasivos e de fácil manuseio.

A preocupação com a beleza e a saúde está presente no cotidiano das pessoas, que buscam procedimentos que atendam às suas necessidades e tragam resultados satisfatórios. Os profissionais que lidam com a estética devem ter sensibilidade, serem capacitados para realizar tais procedimentos com excelência e qualidade, com métodos e técnicas apropriados e visando sempre manter a saúde do paciente. 


\section{REFERÊNCIAS}

ALMA, Jeanete Moussa; COSTA, Magda Lucy Ribeiro Botelho da. O mundo midiático no mundo da beleza: como as esteticistas adquirem os seus produtos cosméticos. Rumores-Revista de Comunicação, Linguagem e Mídias, v. 5, n. 2. 2011. BECKER, Guilherme Kunzler. Assistência Farmacêutica em Estética. 2012. BORGES, Fábio dos Santos; SCORZA, Flávia Acedo. Fundamentos decriolipólise. Fisioterapia Ser, São Paulo, v. 9, n. 4, p.219-224, dez. 2014.

BORTOLOZO, Fernanda; BIGARELLA, Roberto Luis. APRESENTAÇÃO DO USO DE FIOS DE POLIDIOXANONA COM NÓS NO REJUVENESCIMENTO FACIAL NÃO-CIRÚRGICO. Brazilian Journal Of Surgery And Clinical Research, Porto Alegre, v. I6, n.3, p.67-75, nov. 2016.

BRASIL. Conselho Federal de Farmácia. Resolução no 645 de 27 de Julho de 2017. Dá nova redação aos artigos $2^{\circ}$ e $3^{\circ}$ e inclui os anexos VII e VIII da Resolução/CFF no 6i6/15. Diário Oficial da União.

BRASIL. Conselho Federal de Farmácia. Resolução nº 573 de 22 de maio de 2013. Dispõe sobre as atribuições do farmacêutico no exercício da saúde estética e da responsabilidade técnica por estabelecimentos que executam atividades afins.

BRASIL. Conselho federal de farmácia. Resolução no 6I6, de 25 de novembro de 2015. Diário Oficial da União. Brasília-DF. 2015

CABEDA, S. T. L. Uma estranha no espelho: feminilidade, imagem corporal e envelhecimento na contemporaneidade. Sitientibus, Feira de Santana, n. 4I, p. 195-209, dez. 2009 .

CAMARGO, Brigido Vizeu et al. Representações sociais do corpo: Estética e Saúde. Temas em Psicologia, v. 19, n. I, p. 257-268. 2011.

COLHADO, Orlando Carlos Gomes; BOEING, Marcelo; ORTEGA, Luciano Bornia. Mecanismo de ação responsável pela toxicidade do botuToxina Botulínica no Tratamento da Dor. Rev Bras Anestesiol, Santa Catarina, v. 59, n. 3, p.366-38I, maio 2009. 
CORREAA, Michele Santos et al. Análise da eficácia da carboxiterapia na redução do fibro edema gelóide: estudo piloto. Fisioterapia Ser, Belo Horizonte, v. 2, n. 3, p.79-82, maio 2008.

CUNHA, M.B. Peeling Químico: Preparações Farmacêuticas para a Renovação Celular, Alegre/ES, 2014.

GUERRA F. M. R. M.; KRINSK G.G.; CAMPIOTTO L.G.; GUIMARÃES K.M.F. Aplicabilidade dos Peeling Químicos em Tratamento Faciais. Brazilian Journal of Surgery and Clinical Research - BJSCR. Vol.4,n.3, pp.33-36 (Set-Nov 2013) HIRATUKA, C. et al. Relatório de Acompanhamento Setorial - Cosméticos. ABDI Agência Brasileira de Desenvolvimento Industrial. Maio de 2008. v.I.

Leal, VCLV. et al. Cultura da beleza: corpo ideal, cirurgia estética e a promoção da saúde de jovens universitários. Fortaleza, 2007. 87 p. Dissertação (Mestrado) Universidade de Fortaleza 200.

LORENZET, Anelise Regina Regina et al. UMA ABORDAGEM DOS ASPECTOS LEGAIS PARA ABERTURA DE UMA CLÍNICA DE BIOMEDICINA ESTÉTICA. Temiminós Revista Científica, v. 5, n. 2, p. 28-45, 2015.

PINTO R. F. O Uso do Peeling Químico e Mecânico. Revista Pesquisa em Saúde. CDD. 2i ed. 6I3. P II-I6. Belém/ Pará, 20I2.

SERAFIN, Claudia; CORREIA JÚNIOR, Daniel; VARGAS, Mirella. Perfil do farmacêutico no Brasil: relatório. Brasília: Conselho Federal de Farmácia, 2015.

SILVA, Jane Guiname Mota; FILONI, Eduardo; FITZ, Fátima Faní. Fisioterapia no tratamento das disfunções estéticas corporais-revisão de literatura. Manual Therapy, Posturology \& Rehabilitation Journal, v. I2, p. 979-1012, 2014.

SOUSA, Poliana Mendonça de. A atuação do biomédico em procedimentos estéticos não invasivos e invasivos não cirúrgicos. Brasília: 2012.

SHMIDTT, Alexandra; OLIVEIRA, Claudete; GALLAS, Juliana Cristina. O mercado da beleza e suas consequências. UNIVALI, Balneário Camboriú, Santa Catarina, 2008. 